\title{
Dermatofibrosarcoma protuberans associated with trauma: A case report
}

\author{
ROBYN CABRAL ${ }^{1}$, MATTHAUS WILFORD ${ }^{1}$ and MICHAEL J. RAMDASS ${ }^{2,3}$ \\ ${ }^{1}$ Department of Intensive Care and Anaesthetics, Arima General Hospital; ${ }^{2}$ Department of Surgery, \\ General Hospital, Port-of-Spain; ${ }^{3}$ Department of Clinical Surgical Sciences, Faculty of Medical Sciences, \\ University of The West Indies, St. Augustine Campus, Trinidad, West Indies
}

Received August 23, 2019; Accepted June 19, 2020

DOI: $10.3892 / \mathrm{mco} .2020 .2121$

\begin{abstract}
A case of dermatofibrosarcoma protuberans (DFSP) in a 22-year old female associated with blunt trauma of the mid-upper back is presented in the current study with a review of the literature. DFSP is a rare slow growing sarcoma of the soft tissue most commonly seen on the trunk and upper extremities with a low to intermediate grade malignant potential, high chance of local invasion and a high local recurrence rate. The literature search revealed that both non-congenital mutation as well as trauma serve a role in the development of this dermal neoplasm, but the exact mechanism by which trauma may predispose to development of DFSP is unknown. However, it seems intuitive that chronic inflammation and stimulation of the immune system at a local level may trigger the immunopathologic changes that could lead to the malignant transformation of dermal cells. Future research into the relationship between trauma and DFSP on a cellular level is required, as there appears to be a direct link present.
\end{abstract}

\section{Introduction}

Dermatofibrosarcoma protuberans (DFSP) is a rare slow growing sarcoma of the dermis, with a low propensity for distant metastases but high likelihood of local invasion into subcutaneous tissues. As a result, this tumor is treated with wide local excision, ensuring negative resection margins. Recurrence is a feature of DFSP when excision is incomplete. DFSP is most commonly seen in young adults on the trunk and limbs.

Fibrosarcomatous transformation sometimes occurs in a subset of DFSP tumours, referred to as FS-DFSP. Accurate differentiation of this variant is associated with a worse prognosis due to the incidence of distant metastases as compared to ordinary DFSP. Although the aetiology remains unclear,

Correspondence to: Professor Michael J. Ramdass, Department of Surgery, General Hospital, Port-of-Spain, Charlotte Street, Trinidad, West Indies

E-mail: jimmyramdass@gmail.com

Key words: dermatofibrosarcoma protuberans antecedent trauma has been proposed as a predisposing factor in the development of DFSP $(1,2)$.

\section{Case report}

A 22-year old female presented with a history of sharp sensations, described as 'sticking' in nature over the mid-back region. Two years later the patient had a fall and sustained blunt trauma to her back. She then noted that the area became swollen approximately $4 \mathrm{~cm}$ and was painful. Swelling of the area gradually decreased, however the sharp pain persisted. There was no further medical intervention at this time. Four years later, the area in question began to increase in size once again, this time without any precipitating factors. Within two months the lesion grew to approximately $4 \mathrm{~cm}$ and was accompanied by return of excruciating pain with spontaneous resolution of swelling within one month. One-year later the lesion returned however, now painless, and grew to $8 \mathrm{~cm}$ within 4 months (Fig. 1). No Erythema nor discharge were noted at this time. The area became pruritic and developed overlying telangiectasia. The social history revealed occasional alcohol use with no smoking.

The mass was completely excised with a wide margin and a rotational skin flap done to cover the large defect (Figs. 2 and 3). Histological evaluation revealed a well-circumscribed non-encapsulated highly cellular fibroblastic proliferation. Cells displayed scant cytoplasm, tapering elongated nuclei with granular chromatin. Abnormal mitoses were seen at a rate of 10 per HPF. Immunohistochemical analysis showed the tumor to be SMA, Desmin, CD 34, and S100 negative with Ki-67: $30 \%$ proliferation. No vascular or local invasion was present.

\section{Discussion}

DFSP is a rare tumor of the dermis with unknown etiology. The first case of DFSP was described by Taylor in 1890 (3). It was first described as a distinct cutaneous disease entity called progressive and recurring dermatofibroma by Darier and Ferrand in 1924. DFSP is a slow growing sarcoma with a low likelihood of distant metastases, but can often result in local invasion of neighboring tissues. As a result, wide and deep local surgical excision has been the mainstay method of 
treatment. However, even 3-cm-wide local excision margins have resulted in a local recurrence rate of $11 \%$ and as such, new methods have been employed in management. In recent years, reports of DFSPs successfully treated with Mohs micrographic surgery have been appearing in the literature (4).

DFSP most commonly presents on the trunk $(50 \%)$ and extremities (30-40\%) with 10-15\% of cases involving the head and neck (5). The overall annual incidence has been found to be 4.2 per million per year worldwide (6). According to Scott et al (7) the incidence in the Afro-Caribbean population is almost double that of the Caucasian (6.5 and 3.9 per million respectively). The most common age of presentation is between the ages of 30 and 50 and throughout our search of the literature, it was found that the prevalence or DFSP in the male gender outweighed that of the female. Our index case fell outside of these margins with a 22-year old female. One study by Criscione and Weinstock, contradictorily found that females were more frequently affected by DFSP (6).

DFSP is associated with translocation of genetic material between chromosomes 17 and 22 . This translocation $(17 ; 22)$ fuses the COL1A1 gene on chromosome 17 with part of PDGFB gene on chromosome 22 . These mutations which occur after birth, are not inherited and are only found in tumor cells (2). Two types of DFSP have been described; $85 \%$ of these display a more indolent course and have low potential for metastases, representing the classical DFSP. The remaining $15 \%$ are described as fibrosarcomatous 'high grade' type (FS-DFSP), and these can present more aggressively (1).

Benign fibrohistocytomas (BFH) are slow growing, asymptomatic neoplasms of the dermis which usually measure less than $1 \mathrm{~cm}$ in diameter (8). Like DFSP, these lesions show spindle shaped cells during microscopic evaluation. BFH is usually differentiated from DFSP by the positive finding of CD34 in the latter. However, not all DFSP are CD34 positive. A very small percentage may be negative for this immunohistochemical marker as documented by Fernandez-Flores and Manjon (9). Despite negative staining of our index case for CD34, we still lean toward the diagnoses of DFSP versus BFH due to the presence of features such as overlying venous dilatation, large size and rapid increase in size over short period of time, more in keeping with a clinical diagnosis of DFSP as opposed to BFH. Although other Immunohistochemical markers used to differentiate between these two neoplasms are CD10 Apo D FXIIIa and NGFR, due to lack of resources, we were unable to check for the presence of the above mentioned in this case.

In the case described above, the area of skin at which DFSP subsequently developed, displayed altered sensation prior to sustaining blunt trauma. Considering this sensory change, we hypothesize that a pathological process possibly involving incidental translocation $(17 ; 22)$, had begun at the site prior to injury. With subsequent trauma and release of inflammatory mediators, propagation of the malignant process may have occurred, thus adding insult to injury and resulting finally in development of a rapidly growing DFSP. A similar inference has been made by Boukovalas et al, who suggest that trauma and dermal debris causing chronic low grade infection and inflammation, may trigger the molecular changes that lead to development of DFSP. They explained that the exact mechanism by which trauma may predispose to development of DFSP is unknown, but it seems intuitive that

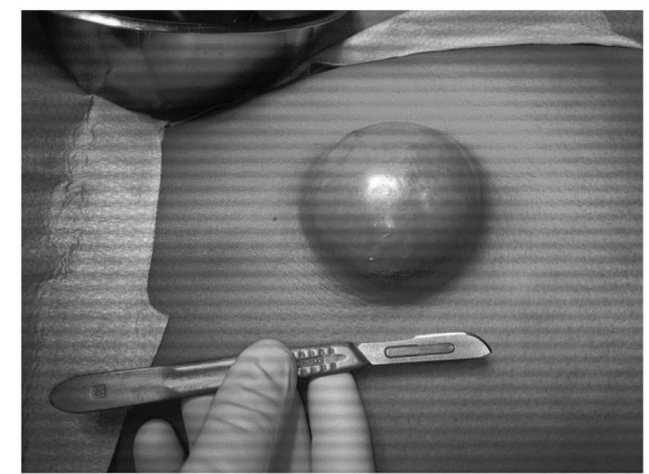

Figure 1. An $8 \mathrm{~cm}$ DFSP on the mid-back of a 22 -year old female. DFSP, dermatofibrosarcoma protuberans

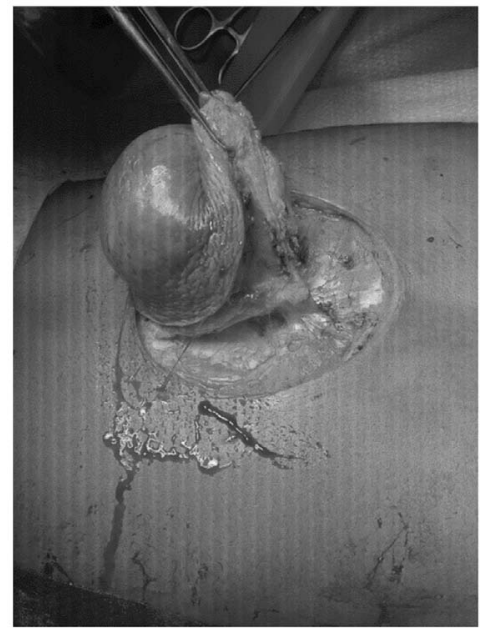

Figure 2. Excision of DFSP with a wide margin. DFSP, dermatofibrosarcoma protuberans.

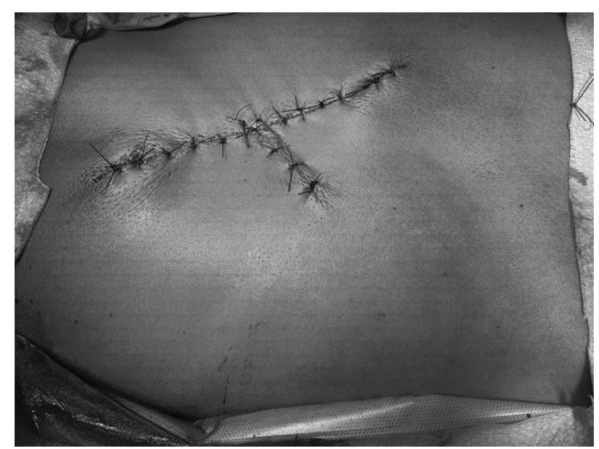

Figure 3. Coverage of large defect using rotational skin flap.

chronic inflammation and stimulation of the immune system at a local level may trigger the immunopathologic changes that could lead to the malignant transformation of dermal cells. A similar mechanism has been described in the pathogenesis of Marjolin ulcers after trauma or skin injury (1). Of note, no research linking inflammatory mediators or other molecular effects of trauma on tissues, to development of malignant processes, was found during our literature search.

Throughout the literature, however, DFSP has been reported to arise in areas with history of prior trauma, 
including tattoos, vaccination sites, burn scars, surgical scars and radiation treatment, trauma scars, radiodermatitis, sites of central venous lines and insect bites $(1,10)$. According to Monnier et al, in a study conducted, $21 \%$ of patients had a history of prior trauma at the site where DFSP developed (11). Of note, all cases linked to trauma found in the literature involved some form of breech of skin or penetrating injury. This is the first case, to our knowledge, documented to show development of DFSP after blunt trauma.

Traditionally, DFSP has been described as a slow growing tumor, but our case was an exception to this description. The tumor grew from 0 to $8 \mathrm{~cm}$ within 4 months. Few similar cases were described in the literature.

Limitations in this case included the lack of IHC markers needed for complete diagnosis of the tumor excised. This lack of resources poses a hindrance to diagnosis of many tumors in our setting.

Here we have described a case of suspected DFSP of the trunk with a history of trauma, which presented on an area of skin with previous altered sensation, in a young female.

Based on the literature search and our current findings, the authors conclude that both non congenital mutation as well as trauma play a role in the development of this dermal neoplasm. Future research into the relationship between trauma and DFSP on a cellular level is suggested, as there seems to be direct link.

\section{Acknowledgements}

Not applicable.

\section{Funding}

No funding was received.

\section{Availability of data and materials}

Not applicable.

\section{Authors' contributions}

$\mathrm{RC}$ was involved in conception and design, acquisition of data, drafting the manuscript and revising it critically for important intellectual content. MW was involved in substantial contributions to conception and design and drafting the manuscript. MJR was the operating surgeon, was responsible for the conception of this paper and revised it critically for important intellectual content. All authors have read and given final approval of the version to be published.

\section{Ethics approval and consent to participate}

Both verbal and retrospective written consent were provided by the patient whose information was used for the writing of this case. In addition, consent for journal publication was also obtained from the patient.

\section{Patient consent for publication}

Consent was received from the patients owing to the publication of data.

\section{Competing interests}

The authors declare that they have no competing interests.

\section{References}

1. Boukovalas S, Castillo AC, Andry D, Lombana N, Qiu S and Murphy KD: Dermatofibrosarcoma Protuberans: Trauma and genetics. Ann Plast Reconstr Surg 1: 1001, 2017.

2. Patel KU, Szabo SS, Hernandez VS, Prieto VG, Abruzzo LV, Lazar AJ and López-Terrada D: Dermatofibrosarcoma protuberans COL1A1-PDGFB fusion is identified in virtually all dermatofibrosarcoma protuberans cases when investigated by newly developed multiplex reverse transcription polymerase chain reaction and fluorescence in situ hybridization assays. Hum Pathol 39: 184-193, 2008.

3. Garg MK, Yadav MK, Gupta S, Kumar N and Khandelwal N: Dermatofibrosarcoma protuberans with contiguous infiltration of the underlying bone. Cancer Imaging 9: 63-66, 2009.

4. Haycox CL, Odland PB, Olbricht SM and Casey B: Dermatofibrosarcoma protuberans (DFSP): growth characteristics based on tumor modeling and a review of cases treated with mohs micrographic surgery. Ann Plast Surg 38: 246-251, 1997.

5. Stamatakos M,Fyllos A, Siafogianni A, Ntzeros K, Tasiopoulou G, Rozis M and Kontzoglou K: Dermatofibrosarcoma protuberans: A rare entity and review of the literature. J BUON 19: 34-41, 2014.

6. Criscione VD and Weinstock MA: Descriptive epidemiology of dermatofibrosarcoma protuberans in the United States, 1973 to 2002. J Am Acad Dermatol 56: 968-973, 2007.

7. Scott N, Causey C, Hodder SC and Kittur MA: 'It Started as a Spot' ... Dermatofibrosarcoma Protuberans. J Cytol Histol S5: 1, 2016.

8. Vanni R: Skin: Cutaneous benign fibrous histiocytomas. Atlas Genet Cytogenet Oncol Haematol 5: 213-214, 2001.

9. Fernandez-Flores A and Manjon JA: Mitosis in dermatofibroma: A worrisome histopathologic sign that does not necessarily equal recurrence. J Cutan Pathol 35: 839-842, 2008.

10. Stivala A, Lombardo GA, Pompili G, Tarica MS, Fraggetta F and Perrotta RA: Dermatofibrosarcoma protuberans: Our experience of 59 cases. Oncol Lett 4: 1047-1055, 2012.

11. Monnier D, Vidal C, Martin L, Danzon A, Pelletier F, Puzenat E, Algros MP, Blanc D, Laurent R, Humbert PH and Aubin F: Dermatofibrosarcoma protuberans: A population-based cancer registry descriptive study of 66 consecutive cases diagnosed between 1982 and 2002. J EurAcad Dermatol Venereol 20: 1237-1242, 2006. 